\title{
Prevalence of Electrocardiographic Abnormalities Among People Living With HIV/AIDS in Sub-saharan Africa: Protocol for a Systematic Review and Meta- analysis
}

Andrew Weil M Semulimi ( $\square$ andrewweil89@gmail.com )

Makerere University https://orcid.org/0000-0003-0588-1559

Andrew Peter Kyazze

Makerere University CHS: Makerere University College of Health Sciences

John Mukisa

Makerere University CHS: Makerere University College of Health Sciences

Charles Batte

Makerere University CHS: Makerere University College of Health Sciences

Felix Bongomin

Gulu University Faculty of Medicine

\section{Protocol}

Keywords: Electrocardiogram, HIV/AIDS, Cardiovascular diseases

Posted Date: August 2nd, 2021

DOI: https://doi.org/10.21203/rs.3.rs-743165/v1

License: (c) (1) This work is licensed under a Creative Commons Attribution 4.0 International License.

Read Full License 


\section{Abstract}

Introduction

Electrocardiographic (ECG) abnormalities are highly prevalent among people living with HIV/AIDS (PLWHA) contributing to the rising burden of cardiovascular diseases among this population. SubSaharan Africa (SSA) contributes more than two thirds to the global burden of HIV/AIDS. However, an accurate account of the prevalence of ECG abnormalities in SSA is unknown. This study aims to determine the prevalence of ECG abnormalities among PLWHA in Sub-Saharan Africa.

Methods and analysis

A systematic review and meta-analysis will be conducted. Databases including EMBASE, MEDLINE, African Journal Online and The Cochrane Library (Cochrane Central Register of Controlled Trials (CENTRAL), and Cochrane Methodology Register) will be used to search for studies published between $1^{\text {st }}$ January 2000 and $31^{\text {st }}$ December 2020. Studies reporting any form of ECG abnormalities will be considered. Additionally, articles in both English and French will be reviewed. Articles will be screened and reviewed by two independent reviewers to determine their eligibility and any disagreement will be resolved through discussions with fourth and fifth reviewer. Eligibility of the studies will be assessed and judged based on the pre-set criteria. Retrieved studies will be assessed for validity using the Cochrane risk of bias tool and the modified Newcastle Ottawa Scale by two reviewers. Analysis of proportions will be done using STATA MP Version 16.0. A p $<0.05$ will be considered statistically significant.

\section{Discussion}

In the face of a growing burden of cardiovascular diseases among PLWHA, this study will provide an estimate of ECG abnormalities among PLWHA which will emphasize the importance of ECGs in screening for cardiovascular diseases in this population.

Systematic review registration number

CRD42021243664.

\section{Introduction}

As a result of anti-retroviral therapy (ART), the life expectancy of PLWHA has increased significantly from 31.3 years and 38.6 years in the 1990 s to 56.1 years and 51.4 years in the 2010 s among women and men respectively as a result of Anti-retroviral therapy (ART) (1). This has led to an increment in the non-HIV related mortality and morbidity (2). CVDs are becoming of great importance in this population with a majority of PLWHA at a risk of developing heart failure, and electrocardiographic abnormalities (3-5). ECG abnormalities are prevalent among PLWHA (5) due to chronic inflammation (6), low CD4 count, high HIV RNA viral load, and duration of ART $(5,7)$ as well as the increasing prevalence of traditional risk factors such as older age, smoking, alcohol use, coronary artery disease, and congestive heart failure (7). 
Electrocardiographic (ECG) abnormalities are widely prevalent and significantly contribute to the cardiovascular disease (CVDS) mortality and morbidity globally, which poses a serious public health challenge (8). The number of CVDS has nearly doubled over three decades with close to 18.6 million deaths in 2019 worldwide an increase of 6.5 million over the 30-year period (8). Compared to high-income countries, Sub-Saharan Africa (SSA) contributes close to six percent of the global mortality resulting from CVDs with ischemic heart disease being the lead contributor closely followed by cerebrovascular disease (4). Cardiac rhythm abnormalities (arrhythmias) that could lead to hemodynamic disturbances causing cerebrovascular accidents, heart failure, or death $(7,9-12)$ are common among the ageing population $(8,13,14)$. The number of prevalent cases with atrial fibrillation and flutter, a common ECG abnormality, were estimated at 59.7 million in 2019 worldwide (8). Additionally, the prevalence of major ECG abnormalities ranged from $2.37 \%$ to $11.3 \%$ and $1.04 \%$ to $12.9 \%$ in men and women respectively (1519). Old age and pre-existing CVDS have been associated with an increase in the prevalence of ECG abnormalities $(15,20,21)$. With an increase in the HIV ageing population (22), the rise in ECG abnormalities among the elderly presents a conundrum for health care providers.

An ECG is a non-invasive, cheap, and widely available diagnostic tool used in the early identification of CVDs such as arrhythmias and is highly predictive of incident CVDs $(5,23)$. It is therefore an important tool which could be scaled up for use in rural health facilities and can be used for early diagnosis of CVDs in PLWHA thus contributing to the reduction of CVD related deaths and morbidity in PLWHA.

The prevalence of ECG abnormalities among HIV positive populations has been estimated between $50 \%$ and $93 \%(5,23-25)$. Since the data may not be applicable to other Africa settings, the accurate estimate of the burden of ECG abnormalities in SSA is unknown. We hypothesize that SSA has a high prevalence of variant ECG abnormalities among PLWHA. Therefore, this study aimed to establish the prevalence of ECG abnormalities among PLWHA in SSA.

\section{Methods And Analysis}

The systematic review and meta-analysis will be done in accordance to the Preferred Reporting Items for Systematic Reviews and Meta-Analyses (PRISMA) checklist (26) (supplement 1).

\section{Search strategy:}

With the aid of a medical librarian, a search will be conducted through EMBASE, MEDLINE, and The Cochrane Library (Cochrane Central Register of Controlled Trials (CENTRAL), and Cochrane Methodology Register) databases for studies published online from January 2000 to December 2020. The search terms that will be used are:

\section{ECG abnormalities}

"cardiac arrhythmia" OR "irregular heart rhythm" OR "ECG abnormalities" OR "ECG presentations" OR " atrial fibrillation" OR "QT prolongation" OR "Ventricular conduction defects" OR "QT prolongation index" 
OR "left ventricular hypertrophy" OR "Major AV conduction abnormalities" OR "major isolated ST/T abnormalities" OR "Left axis deviation" OR "Right axis deviation" OR "Major Q/QS waves abnormalities" OR "Minor Q/QS with ST/T abnormalities" OR "Major isolated ST/T abnormalities" OR "High R-waves" OR "Minor isolated ST/T" OR "ST elevation" OR "Incomplete bundle branch block" OR "Minor QT prolongation index" OR "Short PR interval" OR "Right axis deviation" OR "Frequent ventricular premature beats" OR "ventricular arrhythmias" OR "atrial flutter" OR "cardiac conduction delay".

\section{HIV/AIDS}

"Human immune deficiency virus" OR "HIV" OR "people living with HIV/AIDS" OR "PLWHA" OR "PLWHA" OR "PLWHA" OR "acquired immune deficiency syndrome" OR "AIDS".

\section{Sub-Saharan Africa}

"Africa South of Sahara" OR "sub-Saharan Africa" OR "Angola" OR "Benin" OR "Botswana" OR "Burkina Faso" OR "Burundi" OR "Cameroon" OR "Cape Verde" OR "Central African Republic" OR "Chad" OR "Comoros" OR "Democratic Republic of Congo" OR "Cote d'Ivoire" OR "Eritrea" OR "Eswatini" OR "Ethiopia" OR "Gabon" OR "Gambia” OR "Ghana” OR "Guinea” OR “Guinea-Bissau” OR “Kenya” OR "Lesotho" OR "Liberia" OR "Madagascar" OR "Malawi” OR "Mali" OR "Mauritania” OR “Mauritius" OR "Mozambique” OR "Namibia" OR "Niger" OR "Nigeria" OR "Rwanda" OR "Sao Tome and Principe" OR "Senegal" OR "Seychelles" OR "Sierra Leone" OR "Somalia” OR "South Africa” OR "South Sudan" OR "Sudan” OR "Tanzania” OR “Togo" OR “Uganda” OR "Zaire” OR "Zambia” OR "Zimbabwe”.

In addition, a manual literature search of all cited articles in these papers will be done. Published articles in English or French will be considered. Google translate will be used to translate articles in French.

\section{Study Selection criteria}

Inclusion criteria:

- Studies reporting on any form of ECG abnormality among HIV positive individuals in SSA will be considered from 2000 to 2020 .

- Published articles conducted as observational cross-sectional, case-controls, retrospective studies, case series, case reports, longitudinal studies and randomized clinical trials will be included.

Exclusion Criteria

- Systematic reviews, editorials will be excluded.

\section{Data extraction and management.}

An excel sheet will be designed using Microsoft Excel V.2016 and used to extract the data from the articles. Information such as first authors, year of publication, study country, study design, age (mean or median), sex, the sample size of the participants, number of patients diagnosed with different forms of 
ECG abnormalities, criteria used to define the ECG abnormality, how the diagnosis was made, and the period of data collection in each study will be extracted. Study titles and/or abstracts collected through the search strategy will be screened independently by three authors to identify studies that potentially met the inclusion criteria. Other articles will be retrieved from the reviewed studies provided they meet the criteria. These will be coded by two reviewers who will extract, further verify, and validated the articles. Any variation that may arise will be discussed and resolved by other reviewers. Original authors of published articles with missing or unclear data will be contacted for clarification where possible. All study data will be stored on password protected external hard drives.

\section{Outcomes:}

Prevalence of ECG abnormalities among PLWHA in SSA.

\section{Quality Assessment:}

All the selected studies will be assessed for validity using The Cochrane risk of bias tool (27) and the modified Newcastle Ottawa Scale (28) quality assessment tool by two reviewers. The risk of bias will be graded as low, moderate, or high and if the study had a high risk of bias, it will be excluded from the meta-analysis.

\section{Data analysis}

A random or fixed effect model meta-analysis will be performed using metaprop command for analysis of proportions in STATA 16.0 and presented as prevalence, $95 \% \mathrm{Cls}$ and weights. A forest plot will be used to present the results of the meta-analysis. A systematic descriptive synthesis will be done appropriately to complete the meta-analysis. Heterogeneity of studies will be assessed using Q statistics; $I^{2}$ and $p$-value will be used to report heterogeneity between studies. A $p<0.05$ will be considered statistically significant.

\section{Discussion}

The concise prevalence of ECG changes among PLWHA is unknown. We are unaware of a systematic review and meta-analysis studying the prevalence of ECG abnormalities among PLWHA in Sub-Saharan Africa. Our study will use several data bases to search for articles published over a 20-year period. Our study focuses on the HIV population whose life expectancy has improved tremendously since the introduction of ART in SSA predisposing them to NCDs such as cardiovascular diseases. ECGs play a critical role in the diagnosis and determining the prognosis of patients with suspected cardiovascular diseases. Therefore, results from this study will further highlight the importance of incorporation of comprehensive cardiovascular diseases assessment in the management of PLWHA. This study is limited by the non-uniform reporting of ECG abnormalities.

Our findings will form a basis for the inclusion of ECGs in the screening of PLWHA for cardiovascular diseases. Findings from this study will inform health policy and treatment guidelines development. 
Results will be published in open-access peer reviewed journals. All amendments made will be highlighted in the manuscript.

\section{Abbreviations}

CVDs Cardiovascular diseases

ECG Electrocardiogram

NCDS Non-Communicable diseases

PLWHA People living with HIV/AIDS.

SSA Sub-Saharan Africa

\section{Declarations}

\section{Ethical approval and consent to participate.}

There will be no public or patient participation in the systematic review and meta-analysis. The study is registered with PROSPERO, registration number CRD42021243664.

\section{Consent for publication.}

Not applicable.

\section{Availability of data and materials.}

The datasets used and/or analysed during the current study are available from the corresponding author on reasonable request.

\section{Competing interests}

The authors declare no competing interest.

\section{Funding}

The study received no funding.

\section{Authors' contributions}

AWS and FB developed the idea of the study and drafted the first version of the manuscript and is the guarantor of this systematic review protocol. APK, JM, and CB revised the manuscript. All authors approved the final version of the manuscript.

\section{Acknowledgements}


Special thanks to Dr. Alison Annet Kinengyere for the assistance rendered to the team in developing the search strategy. The author is supported by the Makerere University Non-Communicable Diseases (MAKNCD) Research Training Program: supported by the Fogarty International Centre of the National Institutes of Health under Award Number D43TW011401. The content is solely the responsibility of the authors and does not necessarily represent the official views of the National Institute of Health.

\section{References}

1. Asiki G, Reniers G, Newton R, Baisley K, Nakiyingi-Miiro J, Slaymaker E, et al. Adult life expectancy trends in the era of antiretroviral treatment in rural Uganda (1991-2012). 2016;30(3):487-93.

2. Kiragga AN, Mubiru F, Kambugu AD, Kamya MR, Castelnuovo B. A decade of antiretroviral therapy in Uganda: what are the emerging causes of death? BMC Infect Dis. 2019;19(1):77.

3. Alonso A, Barnes AE, Guest JL, Shah A, Shao IY, Marconi V. HIV Infection and Incidence of Cardiovascular Diseases: An Analysis of a Large Healthcare Database. 2019;8(14):e012241.

4. Yuyun MF, Sliwa K, Kengne AP, Mocumbi AO, Bukhman G. Cardiovascular Diseases in SubSaharan Africa Compared to High-Income Countries: An Epidemiological Perspective. Glob Heart. 2020;15(1):15-.

5. Soliman EZ, Prineas RJ, Roediger MP, Duprez DA, Boccara F, Boesecke C, et al. Prevalence and prognostic significance of ECG abnormalities in HIV-infected patients: results from the Strategies for Management of Antiretroviral Therapy study. Journal of electrocardiology. 2011;44(6):779-85.

6. Wu KC, Zhang L, Haberlen SA, Ashikaga H, Brown TT, Budoff MJ, et al. Predictors of electrocardiographic QT interval prolongation in men with HIV. Heart. 2019;105(7):559.

7. Hsu JC, Li Y, Marcus GM, Hsue PY, Scherzer R, Grunfeld C, et al. Atrial fibrillation and atrial flutter in human immunodeficiency virus-infected persons: incidence, risk factors, and association with markers of HIV disease severity. J Am Coll Cardiol. 2013;61(22):2288-95.

8. Roth GA, Mensah GA, Johnson CO, Addolorato G, Ammirati E, Baddour LM, et al. Global Burden of Cardiovascular Diseases and Risk Factors, 1990-2019: Update From the GBD 2019 Study. Journal of the American College of Cardiology. 2020;76(25):2982-3021.

9. Lugero C, Kibirige D, Kayima J, Mondo CK, Freers J. Atrial fibrillation among the black population in a Ugandan tertiary hospital. International journal of general medicine. 2016;9:191-8.

10. Dries Daniel L, Exner Derek V, Gersh Bernard J, Domanski Michael J, Waclawiw Myron A, Stevenson Lynne W. Atrial fibrillation is associated with an increased risk for mortality and heart failure progression in patients with asymptomatic and symptomatic left ventricular systolic dysfunction: a 
retrospective analysis of the SOLVD trials. Journal of the American College of Cardiology. 1998;32(3):695-703.

11. Mwita JC, Ocampo C, Molefe-Baikai OJ, Goepamang M, Botsile E, Tshikuka JG. Characteristics and 12-month outcome of patients with atrial fibrillation at a tertiary hospital in Botswana. Cardiovasc $\mathrm{J}$ Afr. 2019;30(3):168-73.

12. Tseng ZH, Secemsky EA, Dowdy D, Vittinghoff E, Moyers B, Wong JK, et al. Sudden Cardiac Death in Patients With Human Immunodeficiency Virus Infection. Journal of the American College of Cardiology. 2012;59(21):1891-6.

13. Kornej J, Börschel CS, Benjamin EJ, Schnabel RB. Epidemiology of Atrial Fibrillation in the 21st Century. 2020;127(1):4-20.

14. Priori SG, Blomström-Lundqvist C, Mazzanti A, Blom N, Borggrefe M, Camm J, et al. 2015 ESC Guidelines for the management of patients with ventricular arrhythmias and the prevention of sudden cardiac death: The Task Force for the Management of Patients with Ventricular Arrhythmias and the Prevention of Sudden Cardiac Death of the European Society of Cardiology (ESC)Endorsed by: Association for European Paediatric and Congenital Cardiology (AEPC). European Heart Journal. 2015;36(41):2793-867.

15. Yu L, Ye X, Yang Z, Yang W, Zhang B, on behalf of the China National D, et al. Prevalences and associated factors of electrocardiographic abnormalities in Chinese adults: a cross-sectional study. BMC Cardiovascular Disorders. 2020;20(1):414.

16. De Bacquer D, De Backer G, Kornitzer M. Prevalences of ECG findings in large population based samples of men and women. Heart (British Cardiac Society). 2000;84(6):625-33.

17. Pinto-Filho MM, Brant LCC, Foppa M, Garcia-Silva KB, Mendes de Oliveira RA, Jesus Mendes da Fonseca M. Major Electrocardiographic abnormalities according to the Minnesota coding system among Brazilian adults (from the ELSA-Brasil cohort study). Am J Cardiol. 2017;119.

18. Denes P, Garside DB, Lloyd-Jones D, Gouskova N, Soliman EZ, Ostfeld R. Major and minor electrocardiographic abnormalities and their association with underlying cardiovascular disease and risk factors in Hispanics/Latinos (from the Hispanic community health study/study of Latinos). Am J Cardiol. 2013;112.

19. Liao YL, Liu KA, Dyer A, Schoenberger JA, Shekelle RB, Colette P. Major and minor electrocardiographic abnormalities and risk of death from coronary heart disease, cardiovascular diseases and all causes in men and women. J Am Coll Cardiol. 1988;12.

20. De Bacquer D, De Backer G, Kornitzer MJH. Prevalences of ECG findings in large population based samples of men and women. 2000;84(6):625-33. 
21. Denes P, Larson JC, Lloyd-Jones DM, Prineas RJ, Greenland P. Major and minor ECG abnormalities in asymptomatic women and risk of cardiovascular events and mortality. Jama. 2007;297.

22. Deeks SG, Phillips AN. HIV infection, antiretroviral treatment, ageing, and non-AIDS related morbidity. BMJ (Clinical research ed). 2009;338:a3172.

23. Njoku PO, Ejim EC, Anisiuba BC, Ike SO, Onwubere BJ. Electrocardiographic findings in a crosssectional study of human immunodeficiency virus (HIV) patients in Enugu, south-east Nigeria. Cardiovasc J Afr. 2016;27(4):252-7.

24. Okoye IC, Anyabolu EN. Electrocardiographic abnormalities in treatment-naïve HIV subjects in south-east Nigeria. Cardiovasc J Afr. 2017;28(5):315-8.

25. Menanga AP, Ngomseu CK, Jingi AM, Mfangam BM, Noubiap JJN, Gweth MN, et al. Patterns of cardiovascular disease in a group of HIV-infected adults in Yaoundé, Cameroon. Cardiovasc Diagn Ther. 2015;5(6):420-7.

26. Moher D, Liberati A, Tetzlaff J, Altman DG, The PG. Preferred Reporting Items for Systematic Reviews and Meta-Analyses: The PRISMA Statement. PLoS medicine. 2009;6(7):e1000097.

27. Higgins JP, Altman DG, Gøtzsche PC, Jüni P, Moher D, Oxman AD, et al. The Cochrane Collaboration's tool for assessing risk of bias in randomised trials. 2011;343.

28. Peterson J, Welch V, Losos M, Tugwell PJOOHRI. The Newcastle-Ottawa scale (NOS) for assessing the quality of nonrandomised studies in meta-analyses. 2011.

\section{Supplementary Files}

This is a list of supplementary files associated with this preprint. Click to download.

- completedPRISMAPchecklist.docx 